ARTICLE / INVESTIGACIÓN

\title{
Evaluation of X-ray ionizing radiation on Plasmodium berghei invasion of erythrocytes
}

${ }^{3}$ Cell Biology Department, Simón Bolívar University, Valle de Sartenejas, Caracas, Venezuela

${ }^{4}$ Physics Department, Simón Bolívar University, Valle de Sartenejas, Caracas, Venezuela.

${ }^{3}$ Radiotherapy Service Gurve, La Trinidad. Caracas, Venezuela.

${ }^{4}$ School of Biological Sciences and Engineering, Yachay Tech University, San Miguel de Urcuquí, Ecuador.

Corresponding author: Ispencer@yachaytech.edu.ec

Abstract: Developing new strategies for designing effective vaccines has become a priority for parasitologists worldwide. There is high interest in designing a vaccine against malaria since it is considered one of the most prevalent infectious diseases in the tropics. We evaluated the effects of X-rays irradiation on the erythrocytic stage of Plasmodium berghei ANKA merozoites and schizonts using doses of ionizing radiation ranging between 10 and 300 Gy on parasitized red blood cells (PRBC) to study the attenuating effects of radiation on the merozoites. Parasitic activity diminution was observed starting at $50 \mathrm{~Gy}$, and the dose for complete attenuation was established at 200 Gy, corresponding with a 100\% survival rate of mice. In vivo invasion experiments and immunofluorescence assays (IFA) showed inhibition of merozoite invasion of the host red blood cells (RBC). Nonetheless, immunization with irradiated parasitized red blood cells (IPRBC) was ineffective in protective assays. We perform cytoadherence and inhibition of cytoadhesion assays on irradiated merozoites. The results showed that high irradiation doses caused an unspecific cellular adhesion phenomenon independent of the ICAM-1 and CD36 interaction, which was determined by Cytoadhesion assays. Our results show that, even though X-ray irradiation is an effective method to induce complete parasite attenuation, it might affect the parasite's membrane surface structures triggering unspecific adhesion.

Key words: Malaria vaccine, LINAC X-ray ionizing radiation, Plasmodium berghei, erythrocyte membrane modification.

\section{Introduction}

Malaria is one of the most prevalent infectious diseases in the tropics. It is caused by a protozoan parasite of the genus Plasmodium and is responsible for millions of cases every year. In 2017, 228 million people were diagnosed with malaria, and around 435,000 malaria deaths were reported ${ }^{1}$.

Plasmodium species are protozoan parasites that belong to the phylum Apicomplexa and display a complex life cycle that involves an invertebrate vector (female Anopheles mosquito) and a vertebrate host. In its vertebrate host, the Plasmodium parasite goes through two stages: in the first stage, sporozoites invade hepatocytes and undergo asexual multiplication into merozoites; in the second, merozoites are released into the bloodstream, where they invade erythrocytes. Inside red blood cells (RBC), merozoites reproduce asexually for a second time, forming the merozoite-RBC complex, also called schizont. These merozoite-filled RBCs burst open, releasing more infectious merozoites that rapidly invade uninfected RBCs to repeat their asexual erythrocytic cycle ${ }^{2,3,4}$.

$X$-ray irradiation is one of the most commonly used methods to achieve effective attenuation of the Plasmodium parasite during the design of malaria vaccines ${ }^{3,5}$. Many studies have reported the use of irradiation of Plasmodium sporozoites to achieve parasite attenuation ${ }^{6}$. However, inoculation of radiation-attenuated sporozoites (RAS) provides poor protection against a second challenge with the natural parasite ${ }^{7}$. A study in 2013 by Seder et al. used an intravenous (IV) inoculation with
RAS to achieve a greater immunological response, obtaining the most promising results in fighting malaria reinfection ${ }^{8}$, yet some concerns remain on the IV administration route and the low rate of success obtained in the trials.

Administration of malaria vaccine RTS-S or Mosquirix (RTS, S) has resulted in a 60\% decrease in the disease's clinical manifestations, but meningitis cases have also been reported in African children who have received this vaccine ${ }^{9,10}$.

Researchers have also reviewed candidate proteins belonging to the merozoite stage, and they have been shown to mediate immunity against the invasion of the erythrocyte ${ }^{11}$. However, there are no reports on merozoite attenuation as a strategy for malaria vaccine design. Therefore, this work aimed to determine the effects of high doses of ionizing radiation on the merozoite stage in the Plasmodium berghei-rodent model and assess this approach's potential to determine the effect of irradiation on the parasite's membrane. In particular, we considered the effect of radiation on cell adherence.

\section{Materials and methods}

\section{Plasmodium berghei ANKA strain and in vitro culture}

Ten-week-old female BALB/c mice, bred under pathogen-free conditions, were obtained from the Simón Bolivar

Citation: Nava-Lauson, C.; Spencer, L.M.; Saho-Bohus, L., Davila, J. and Tellkamp, M. Evaluation of X-ray ionizing radiation on Plasmodium berghei invasion of erythrocytes. Revis Bionatura 2022;7(1). 19. http://dx.doi.org/10.21931/RB/2022.07.01.19

Received: 8 August 2021 / Accepted: 25 October 2021 / Published: 15 February 2022

Publisher's Note: Bionatura stays neutral with regard to jurisdictional claims in published maps and institutional affiliations. 
University's Animal Housing Facility and subsequently infected with the lethal strain Plasmodium berghei ANKA. Blood draws were performed on Krebs' glucose solution (KGS) containing heparin (1:20 dilution of Sodium heparin $1000 \mathrm{U.I.} / \mathrm{ml}$ ) and passed through columns of fibrous cellulose powder (CF 11, Sigma ${ }^{\circledR}$ ) to remove leukocytes ${ }^{12,13}$. For schizont purification, parasitized red blood cells (PRBC) were isolated using PercoUl-Krebs gradient ${ }^{12}$. Merozoites and schizonts were evaluated by short culture in vitro as previously described by Spencer and co-workers ${ }^{14}$. The ethical review board approved all animal experiments of Simón Bolívar University.

\section{Parasitological evaluation of radiated and parasitized red blood cells}

We used a VARIAN Clinac Trilogy ${ }^{\circledR} 2100$ model X-ray machine at $6 M V$ to irradiate schizonts using doses between 10 and 300 Grays (10, 30, 50, 80, 100, 120, 200300 Gy). Following the irradiation process, BALB/c mice were inoculated intra-peritoneally (IP) with $5 \times 10^{3}$ Irradiated Parasitized Red Blood Cells (IPRBC). After inoculation, parasitaemia was assessed daily on blood smears obtained from the mice tails, stained with Giemsa stain and viewed under a light microscope at 1000 magnification ${ }^{15}$. All experiments were repeated in triplicate using groups of five BALB/c mice for each irradiation dose.

After two months, the mice that survived inoculation with the IRBCs were infected with the $P$. berghei ANKA lethal strain with $5 \times 10^{3}$ infected RBCs, and their parasitaemia was assessed daily again. All experiments were repeated three times with groups of five BALB/c mice for each irradiation dose.

\section{Indirect immunofluorescence assay}

The effect of irradiation on the merozoite membrane was evaluated using an indirect immunofluorescence assay (IFA) ${ }^{16}$. We used blood smears of mice inoculated with IPRBCs obtained 10 days post-infection and fixed with acetone. Immune sera acquired from experimental mice inoculated with different doses of IPRBC were used as sources for primary antibodies. Afterward, we incubated the samples with FITC-labeled anti-mouse $\lg G\left(\right.$ Sigma $\left.^{\circledR}\right)$ as a secondary antibody, and the smears were observed with an Optika ${ }^{\circledR}$ microscope XDS-3FL model at 400X magnification. Micrographic pictures were acquired using the OptikPro ${ }^{\circledR}$ software.

\section{Indirect enzyme-linked immunosorbent assay}

Enzyme-linked immunosorbent assay (ELISA), indirect type, was performed to evaluate recognition of superficial antigens present on the schizonts in BALB/c mice infected with P. berghei ANKA ${ }^{17}$. Microplates (96-wells, Nunc $\left.{ }^{\circledR}\right)$ were coated with $2 \times 10^{5}$ PRBC per well $(100 \mu \mathrm{L})$ and blocked by the addition of $3 \%$ bovine serum albumin (BSA) in phosphate buffer saline (PBS). Next, hyper-immune sera (HiS) obtained from mice previously inoculated with IPRBC at different doses of radiation $(80,100,200$ and $300 \mathrm{~Gy})$ were added $1: 100$ as a source of primary antibodies and incubated at $37^{\circ} \mathrm{C}$ for 1 hour. Wells were washed three times with washing buffer (PBS-Tween 0.005\%) and samples were incubated with $100 \mu \mathrm{L} /$ well of horseradish peroxidase (HRP)-conjugated goat anti-mouse polyvalent immunoglobulin (IgG; H+L) $\left(\right.$ Sigma $\left.{ }^{\circledR}\right), 1: 1000$ and incubated for 1 hour at $37^{\circ} \mathrm{C}$.

Finally, after washing the wells with PBS-Tween 0.005\%, we added $100 \mu \mathrm{L} /$ well of azino-bis (3-ethylbenz-thiazoline-6-sulfonic acid) (ABTS, Sigma ${ }^{\circledR}$ ) as substrate in $0.05 \mathrm{M}$ phosphate-citrate buffer ( $\mathrm{pH}$ 5.0) containing $0.05 \% \mathrm{w} / \mathrm{v} \mathrm{H}_{2} \mathrm{O}_{2}$. The reaction was stopped by the addition of $50 \mu \mathrm{L} 2 \mathrm{M} \mathrm{H}_{2} \mathrm{SO}_{4}$ per well. The plates were read at $405 \mathrm{~nm}$ in a microplate reader (Bio-RAD i-Mark ${ }^{\circledR}$ ) using the Titer-soft Software Flow to measure the absorbance. Percentages and dilutions were standardized in preliminary tests. The cut-off point was calculated using absorbance (O.D., optical density) \pm 3 standard deviations from sera of 10 uninfected mice (data not shown).

\section{Western blot assay}

The immunodetection of polypeptides in hyperimmune sera obtained from mice previously inoculated with IPRBC was achieved by Western blot assays. We prepared a P. berghei soluble antigen (PbSA) by sonication of purified schizonts suspended in lysis buffer containing 50mM Tris-HCl pH 8.0, 5mM EDTA, 5mM EGTA, 0.05\% Tween 20 and 1\% complete protease inhibitor cocktail (SC-29130, Santa Cruz Biotechnologies ${ }^{\circledR}$ ). The polypeptides of PbSA were separated by SDS-polyacrylamide $(12 \%)$ gels at $100 \mathrm{~V}$ for 1 hour and then eletrophoretically transferred to a nitrocellulose membrane ${ }^{18}$. A pre-stained protein ladder from Invitrogen ${ }^{\circledR}(170-10 \mathrm{kDa})$ was used as marker.

After transfer, blots were blocked by incubation with a 3\% non-fat milk powder solution in PBS, and proteins were detected with 1:100 primary antibodies dilution (hyper-immune sera from mice previously inoculated with IPRBC at 80, 200 and 300 Gy) by incubating for 1 hour. Blots were subsequently incubated with anti-mouse immunoglobulin conjugated to horseradish peroxidase (Sigma ${ }^{\circledR}$ ) at 1:1000 dilution. Antibody binding was detected by incubating in a solution of $3 \mathrm{mg} / \mathrm{ml}$ 4-chloro-1-napthol in methanol, mixed with $50 \mathrm{~mL}$ of $50 \mathrm{mM}$ Tris- $\mathrm{HCl} \mathrm{pH} 7.5$ and $30 \mu \mathrm{L}$ of $30 \% \mathrm{H}_{2} \mathrm{O}_{2}$. The reaction was stopped by washing with $\mathrm{H}_{2} \mathrm{O}^{19}$.

\section{Cytoadhesion and cytoadhesion inhibition assays}

Cytoadhesion assays were performed in vitro on 24-well plates on which VERO cells had been cultured to confluence. Then, $2 \times 10^{5}$ IPRBC with irradiation doses of 80, 120 and 200 Gy were added to each well and cells were allowed to cytoadherence for $2 \mathrm{~h}$ at $37^{\circ} \mathrm{C}^{20}$. RBCs were used as negative control and PRBC as a positive control. Unattached cells were removed by washing with PBS, and attached cells were fixed for $1 \mathrm{~h}$ with $2.5 \%$ glutaraldehyde $\left(\right.$ Sigma $\left.^{\circledR}\right)$. The IPRBC and PRBC were first incubated with the respective hyper-immune sera at 1:10 dilution for 1 hour ${ }^{21}$. Adherent IPRBC, PRBC and RBC on VERO cells were counted under a light microscope in 4 randomly selected fields at 400X magnification (OptikPro ${ }^{\circledR}$ ). To calculate the number of IPRBC that adhere to VERO cells, counts were recorded in 5 different microscope fields, and each assay was performed in triplicate.

\section{Statistical analysis}

Each experimental value is presented as the mean of five replicates \pm standard deviation. Once normality and homogeneity criteria were met, statistical analyses were carried out by ANOVA and Tukey Test using confidence level at 95\%. All statistical analyses were performed using $\mathrm{R}$ in its version 3.2.3.

\section{Results}

\section{Evaluation of IPRBC in experimental protective assays}

The daily monitoring of parasitaemia is shown in Fig. 1, where we assessed the parasitaemia percentage (\%) as a function of days post-infection. Figure $1 \mathrm{~A}$ suggests reducing parasitaemia percentages in peripheral blood as radiation doses increase. Moreover, animals inoculated with IPRBC with 50 Gy survived four days longer than other experimental groups. As 
shown in fig. 1B, treatments with 80, 120 and 200 Gy accomplished complete control of parasitaemia on days 26, 23 and 20 post-infection, respectively. We observed a survival rate of $33.3 \%$ (5 out of 15 mice) for irradiation with $80 \mathrm{~Gy} ; 66.6 \%$ (10 out of 15 mice) for doses of $120 \mathrm{~Gy}$, and 100\% for irradiation with 200 Gy. Statistical analyses with ANOVA and Tukey tests showed no significant differences between control, 10 Gy and 30 Gy treatments. These results showed that the minimum radiation intensity to attenuate Plasmodium berghei schizont was $50 \mathrm{~Gy}$. However, the most efficient attenuation dose was $200 \mathrm{~Gy}$, which resulted in a 100\% survival rate. In the case of a dose of $300 \mathrm{~Gy}$, all mice died around day 11.

The experimental group that survived IRBC infection with 200 Gy were re-infected with the $P$. berghei strain ANKA, and the parasitaemia percentage was monitored daily as shown in figure 2. It is important to note that sixty days after IPRBC inoculation, we did not observe any parasitaemia on surviving mice. Strikingly, none of the experimental animals survived the second immunological challenge with the lethal strain of $P$. berghei ANKA, and all mice died within 18 days post-infection (Fig. 2). Of note, experimental animals previously inoculated with IPRBC with 200 Gy died four days later than those of the control group. These results suggest that immunization with IPRBC did not provide protective immunity.

Immunofluorescence assays showed X-ray treatment has an inhibitory effect on the Plasmodium berghei merozoite invasion to the RBC. Fig. 3 shows control merozoites have invaded the host RBC after 10 days (panel A), while schizonts irradiated with 120 Gy remain outside the RBC (panel B).

\section{Indirect ELISA}

Indirect ELISA experiments were performed to determine the capacity of sera from mice inoculated with IPRBC to recognize naturally occurring $\mathrm{PRBC}$ as antigen. The results are presented in Fig. 4, which plots absorbance vs. different hyper-immune sera used. These results suggest that radiation might cause damages to the RBC membrane, and therefore, the surface antigens recognized by the antibodies are affected. The recognition epitopes for the irradiated RBC are different, and therefore, the antibodies do not recognize the antigen in the natural state of PRBCs without radiating. Fig. 4 shows a gradual decrease in absorbance concerning immune sera from IRBCs; when the radiation increases, sera from IRBC recognized less the antigenic surface of PRBCs.

\section{Western blot assay}

To evaluate the recognition of the antigenic surface by the IPRBC sera, we used PbSA as antigen and hyper-immune sera from experimental mice previously inoculated with IPRBC at different radiation doses. The PbSA polypeptide pattern was recognized by the hyper-immune sera of $P$. berghei infected mice. Antibodies showed 3 separate protein bands with apparent weights of $103 \mathrm{kDa}, 45-49 \mathrm{kDa}$, and $19 \mathrm{kDa}$ (Fig. 5; lane 2). However, the hyper-immune sera of mice inoculated with IPRBC recognized only the $19 \mathrm{kDa}$ polypeptide (Fig. 5; lanes 3, 4 and 5). The blotting also reveals weaker bands for the 45-49 kDa protein of all hyper-immune sera from the IPRBCs with different radiation doses tested.
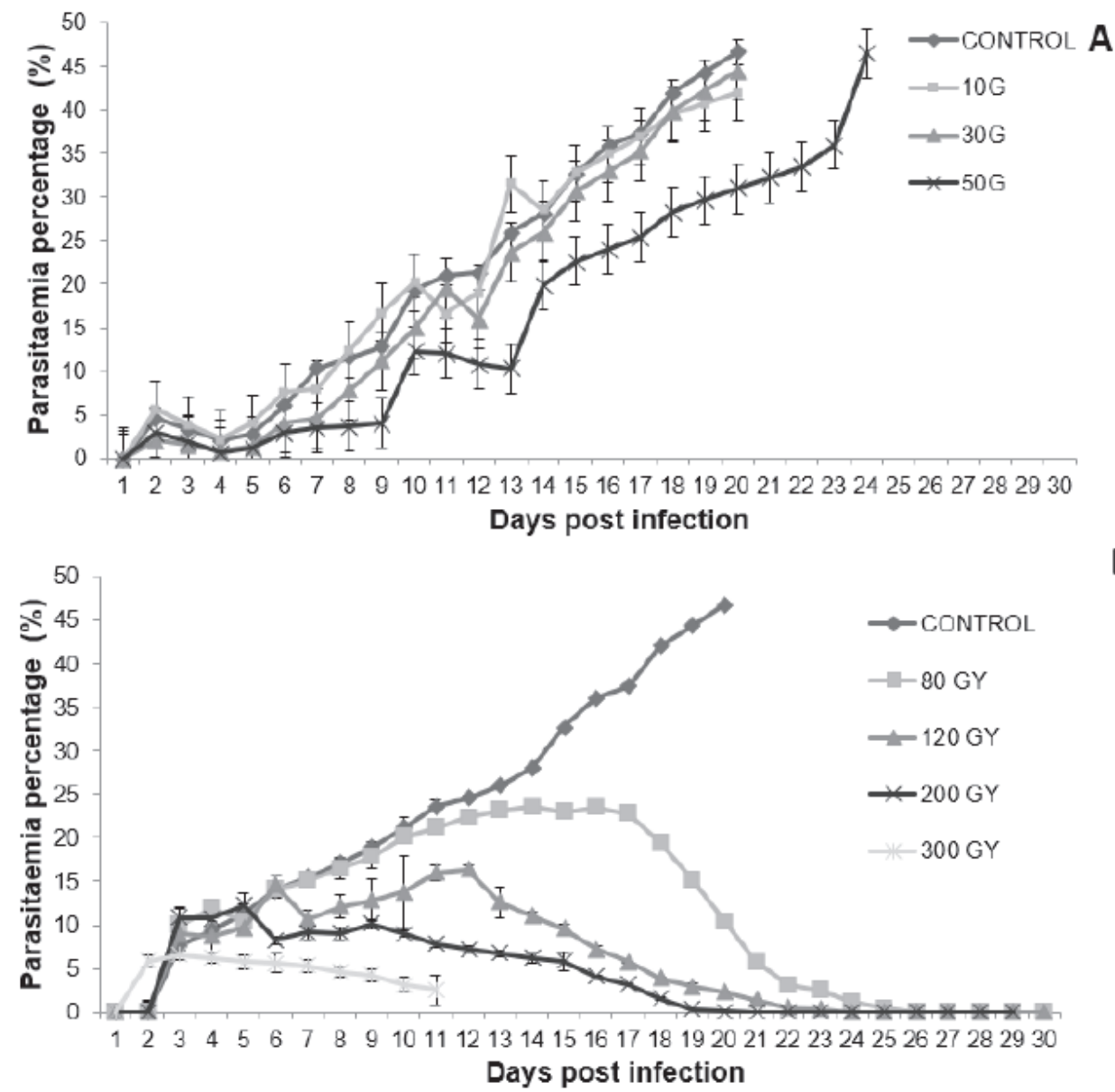

Figure 1. Percentage of parasitaemia observed in BALB/c mice infected with IPRBC, treated with different irradiation doses. We evaluated two independent experiments: A. 10, 30 and 50 Gy, and B. 80, 120, 200 and 300 Gy. The control is the non-irradiated parasites as active infection. Each experiment was assessed with 5x103 IPRBC/mice in three groups of five animals per group. 
MSP-1 is known to be one of the most characteristic proteins on the surface of merozoites and has been described as a potent immune inducer for protective immunity ${ }^{7,22}$. The 45-49 and $19 \mathrm{kDa}$ protein bands recognized by hyper-immune sera correspond to polypeptides associated with the MSP-1 protein after proteolytic cleavage ${ }^{23-26}$. A weaker recognition of the 45$49 \mathrm{kDa}$ protein band suggests that the MSP-1 polypeptide may have undergone alterations in its structure caused by $\mathrm{X}$-ray radiation (lanes 3, 4 and 5). Furthermore, the apparent weight band of $103 \mathrm{KDa}$ was not recognized or very weak by the sera from the different radiation doses.

\section{Cytoadhesion assay in vitro}

We modified the protocol described by Pouvelle and co-workers by using epithelial cells (VERO) instead of endothelial cells to perform the cytoadhesion assays ${ }^{20}$ (See figure 6). Both cell lines (endothelial and Vero) are reported to express the Intracellular Adhesion Molecule 1 (ICAM-1) and CD54; which mediates the adhesion between PRBC and cellular receptors ${ }^{27,28}$. Additionally, epithelial cells express CD36, which acts as a secondary binding molecule for PRBC in natural malarial infections ${ }^{27,28}$

Figure 6 showed a decrease in cytoadhesion for radiations of 80 (98.8 IPRBC / field) and 120 Gy (72.4 IPRBC / field) concerning positive control. Figure 6. E shows that the adhesion levels for schizonts irradiated with 200 Gy (195 IPRBC/ field) are 1.4 times greater than the positive control of parasitized red blood cells (140 GRP / field).

\section{Discussion}

We evaluated the effects of high X-rays doses $(80,100$, 120, 150, 180, 200 and $300 \mathrm{~Gy}$ ) on experimental groups of $\mathrm{BALB} / \mathrm{c}$ mice infected with $5 \times 10^{5} \mathrm{IPRBC}^{14}$. Our results showed a typical parasitaemia curve in positive controls as shown in Fig. 1. The treatment with 120 Gy accomplished the complete control of parasitaemia on the 13th day post-infection, resulting in survival rates over 50\% (8/15). Another relevant observation was that mice inoculated with IPRBC at 300 Gy died around day 11 post-infection with low levels of parasitaemia (between 1.5 and $4 \%$ parasitaemia). This suggests that death is not directly related to malaria infection but to the effect of radiation on IPRBCs. Based on the results obtained from cytoaherence assays (Fig. 6). We suggest this decrease in cytoadhesion is probably due to damage caused by radiation on the cell membranes (for both parasites and RBC). Our hypothesis is supported by the studies of Claessens and Rowe, in which the inhibition of ICAM-1 in Plasmodium falciparum sporozoites causes a decrease in the number of parasites attached to HBEC cells ${ }^{29}$. The observation of IPRBC confirmed this remarkable result aggregates on smears from experimental animals monitored for parasitaemia (data not shown). Consequently, we suggest that death, even at low levels of parasitaemia in peripheral blood, is caused by bloodstream blockage on the microvasculature, indicating a dose-dependent parasite attenuation by ionizing radiation.

IFA show a decrease in merozoite invasion capacity of $\mathrm{RBC}$ in a radiation-dependent manner that correlates with the

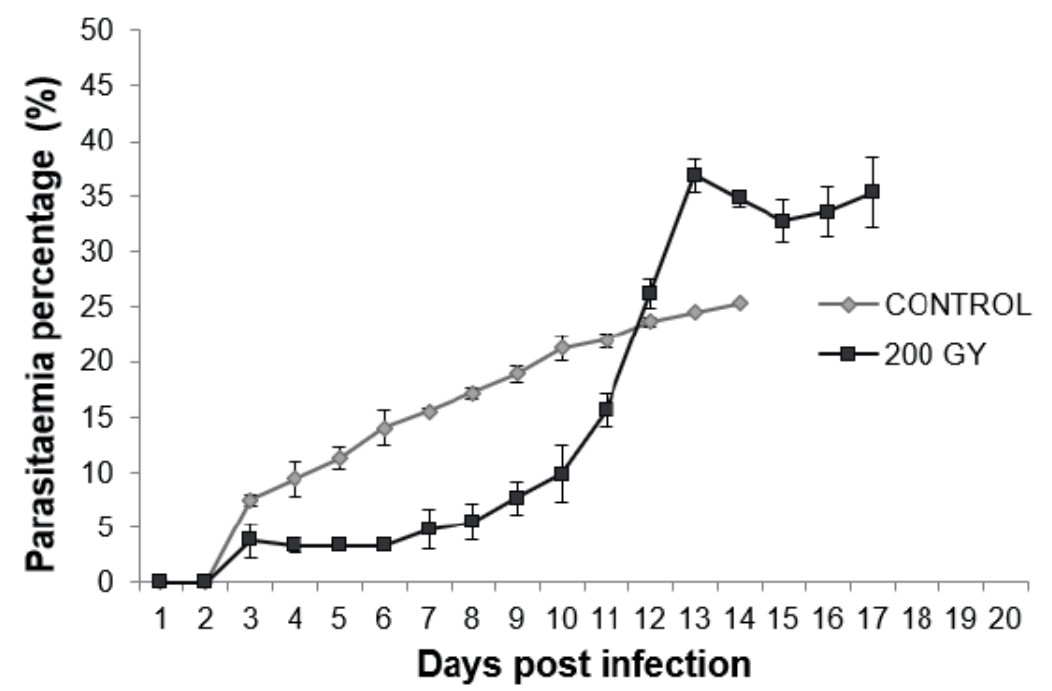

A

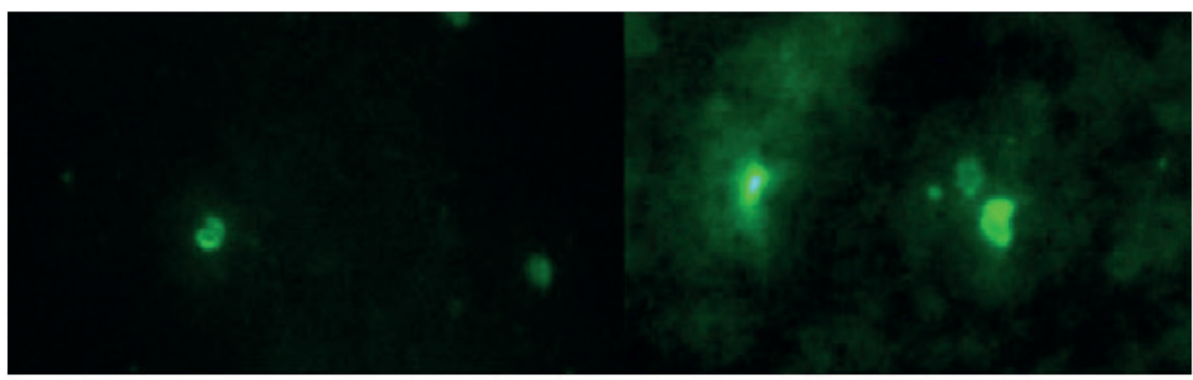

Figure 2. Parasitaemia percentage observed in BALB/c mice re-infected with lethal strain Plasmodium berghei ANKA. For each experiment, mice were inoculated with $5 \times 10^{3}$ parasites/mouse in a control (naïve mice) and 200Gy treatment, using two groups of five animals each.

Figure 3. Immunofluorescence patterns on hyper-immune sera from infected mice; antibodies reactive to MSP-1 were applied to methanol-acetone-fixed smears of erythrocytes infected with $P$. berghei ANKA. A. positive control with PRBC; B. IPRBC with 120 Gy and C. IPRBC with 200 Gy. In panels, B and C show merozoites outside the RBC. 

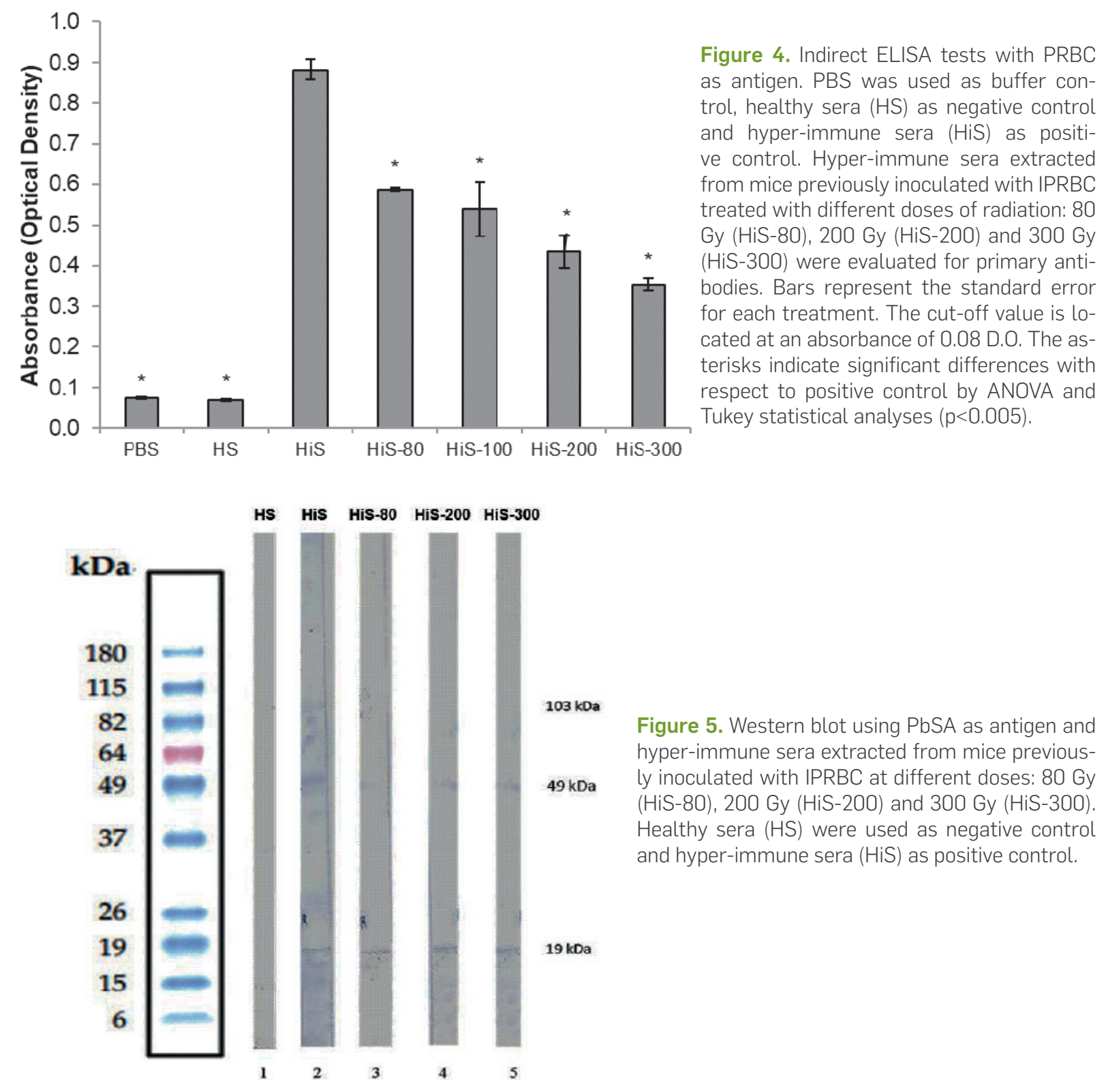

Figure 5. Western blot using PbSA as antigen and hyper-immune sera extracted from mice previously inoculated with IPRBC at different doses: 80 Gy (HiS-80), 200 Gy (HiS-200) and 300 Gy (HiS-300). Healthy sera (HS) were used as negative control and hyper-immune sera (HiS) as positive control.

Low levels of parasitaemia (less than 15\%) circulating in peripheral blood on the 10th day post-inoculation (Fig. 1.B, 120 and 200Gy). We suggest X-rays might alter the conformation of surface antigen as MSP-1 antigen on the parasite's membrane $5,23,24$, which is no longer recognized by antibodies from IPRBC hyperimmune sera as proven by western blot assays (figure 5). Therefore, these results are evidence for the effect of high doses of $\mathrm{X}$-rays on Plasmodium berghei merozoites invasion and suggest that death in experimental animals is not a consequence of malaria infection. An inverse correlation between radiation intensity and absorbance obtained by indirect ELISA suggests $X$-rays may also cause damages on the schizont's membranes and modify the MSP-1 antigen ${ }^{5,7,24}$, thus diminishing the capability of hyperimmune sera to recognize natural PRBC membranes. The absorbance obtained from HiS as a positive control was 0.88 D.O. This value decreases gradually when radiation intensity rises until it reaches a value of 2.5 times lower at HiS-300 (0.35 D.O.) (Fig.4). In consequence, as the radiation applied to PRBC increases, the surface proteins suffer alterations, diminishing their capacity to activate the humoral response ${ }^{30}$. This could explain the changes observed during the

secondary challenge (Fig. 1) and could be the reason for the failure to maintain protection against $P$. berghei ANKA infection.

The results of the cytoadhesion tests are presented in Fig. 6. The black arrows show the number of adhered IPRBCs per field decreases with the treatments of 80 Gy (IPRBC-80) and 120 Gy (IPRBC-120) compared to the positive control (PRBC). Cytoadherence of PRBCs to host cells depends on different proteins that play a relevant role in malaria pathogenicity. Some of those proteins are expressed by the parasite early, such as trophozoite ${ }^{31}$. However, the 200 Gy radiation (IPRBC-200) showed an increase of adhered cells 2 times higher than IPRBC-120, suggesting the adhesion is unspecific and is not mediated by receptors.

\section{Conclusions}

Attenuation of Plasmodium berghei merozoites was observed with X-ray doses starting at $50 \mathrm{~Gy}$, and the effective dose of attenuation was 200 Gy for inoculation with $5 \times 103$ IPRBC/mice resulting in $100 \%$ survival. Moreover, the results 

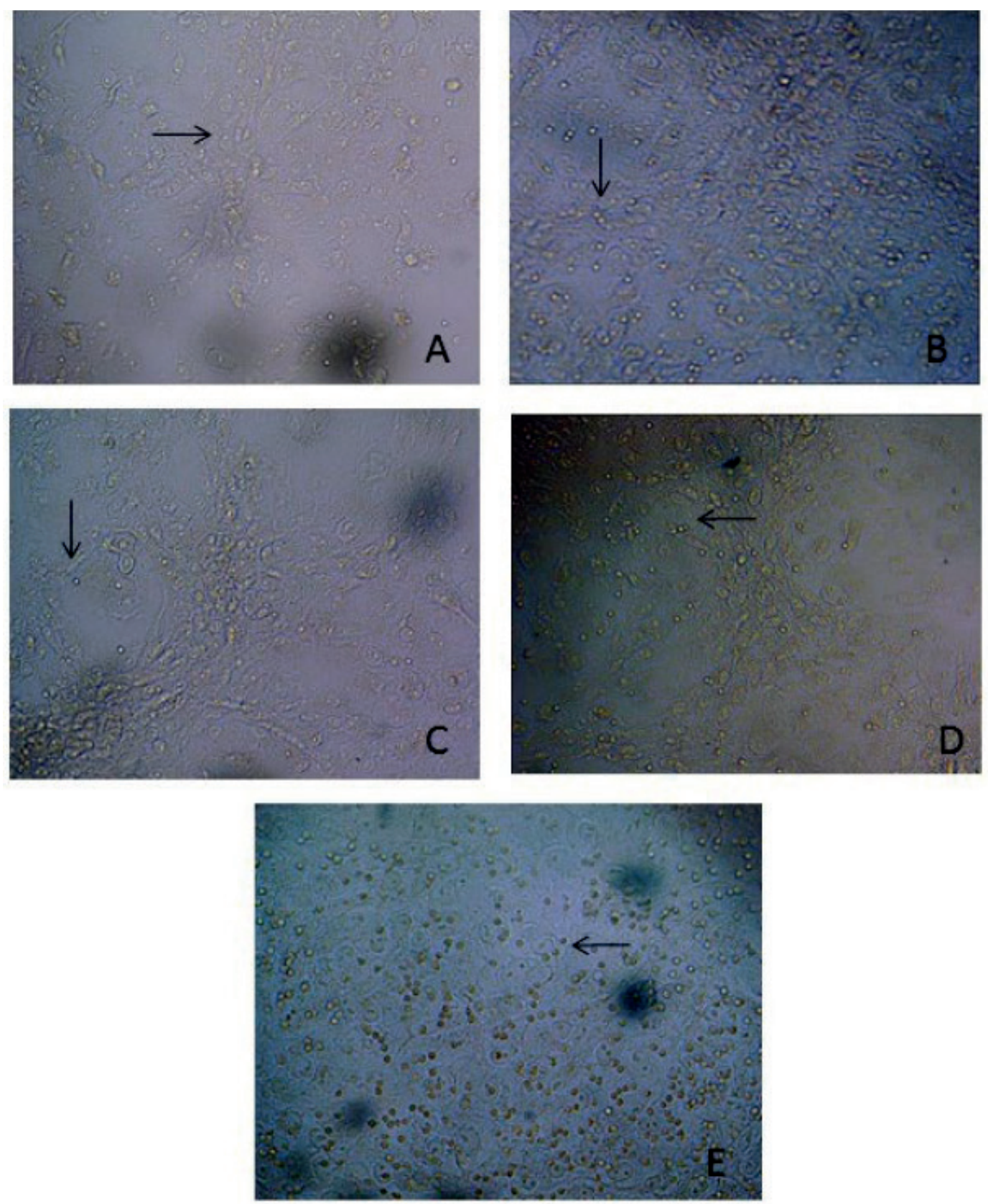

Figure 6. In vitro microagglutination test for IPRBC on VERO cells observed under a light microscope at a magnification of 400X. Panel A corresponds to the negative control, with healthy red blood cells. Panel B is a positive control, PRBCs (parasitized red blood cells) without radiation exposure. Panel $C$ corresponds to IPRBC receiving radiation of 80 Gy. Panel D corresponds to IPRBCs subjected to radiation of $120 \mathrm{~Gy}$, and panel E corresponds to IPRBCs irradiated with a dose of $200 \mathrm{~Gy}$. The arrows point to the parasitized and irradiated RBCs on VERO cells.

of IFAs showed that this effect was caused by an inhibition of merozoite invasion to the host RBC. Nonetheless, this irradiation dose was not effective in protective assays because of the alterations suffered by the membrane structure, as showed in western blot and cytoadhesion tests.

In addition, the cytoadherence results demonstrated that high doses of irradiation cause an unspecific cellular adhesion independent of ICAM-1 and CD36. This unspecific adhesive effect increased in a radiation dependent manner as a possible result of alterations on the integrity of erythrocytic membrane structures. Therefore, this study suggests that radiation of merozoites and schizonts are not an effective strategy for the design of a malaria vaccine.

\section{Author Contributions}

Conceptualization, LMS. CNL and LMS performed all experiments. Irradiation protocols performed by JD. Writing-review and editing, LMS; CNL; LS and MPT. Funding acquisition, LS and LMS. All authors have read and agreed to the published version of the manuscript.

\section{Funding}

This study was financed by a grant from FONACIT (Fondo Nacional de Ciencia, Innovación y Tecnología), No. 294072269, Venezuela.

\section{Institutional Review Board Statement}

The experiments described in this study were carried out 
following the laboratory's standards and the Bioethics Committee of the Simón Bolívar University.

\section{Acknowledgments}

We would like to thank Dr. Juan C. Piña-Crespo, Beatriz Pernia and Sophya Alchali for helpful suggestions and critical reading of the manuscript.

\section{Conflicts of Interest}

The authors declare no conflict of interest.

\section{Bibliographic references}

1. WHO (World Health Organization) [Internet]. World malaria report 2018. [July 2021]. Available from: https://www.who.int/malaria/media/world-malaria-report-2018/en/.

2. Cherfas J. Malaria vaccines: the failed promise. Science. 1990;247(4941):402-403.

3. Carvalho LJM., Daniel-Ribeiro CT, Goto H. Malaria Vaccine: Candidate Antigens, Mechanisms, Constraints and Prospects. Scand J Immunol. 2002;56(4):327-343.

4. Thera MA, Plowe CV. Vaccines for Malaria: How Close Are We? Annu Rev Med. 2012;63(1):345-357.

5. Chattopadhyay R., Conteh S, Li M, James ER, Epstein JE, Hoffman SL. The Effects of radiation on the safety and protective efficacy of an attenuated Plasmodium yoelii sporozoite malaria vaccine. Vaccine. 2009;27(27):3675-3680.

6. Duffy PE, Sahu T, Akue A, Milman N, Anderson C. Pre-erythrocytic malaria vaccines: identifying the targets. Expert Rev Vaccines. 2012;11(10):1261-1280.

7. Purcell LA, Yanow SK, Lee M, Spithill TW, Rodriguez A. Chemical Attenuation of Plasmodium berghei sporozoites induces sterile immunity in mice. Infect Immun. 2008;76(3):1193-1199.

8. Seder RA, Chang LJ, Enama ME, Zephir KL, Sarwar UN, Gordon $\mid J$, et al. Protection against malaria by intravenous immunization with a nonreplicating sporozoite vaccine. Science. 2013:341(6152):1359-1365.

9. Schwartz L, Brown GV, Genton B, Moorthy VS. A review of malaria vaccine clinical projects based on the WHO rainbow table. Malar J. 2012;11(1):11.

10. Sackey AH. Musings on malaria morbidity and mortality after the new Mosquirix ( $\left.{ }^{\circledR}\right)$ vaccine. Ghana Med J. 2019;53(2):87-188.

11. Beeson JG, Drew DR, Boyle MJ, Feng G, Fowkes FJ, Richards JS. Merozoite surface proteins in red blood cell invasion, immunity and vaccines against malaria. FEMS Microbiol Rev. 2016;40(3):343-72.

12. Richards WH, Williams SG. The removal of leucocytes from malaria infected blood. Ann trop med parasitol. 1973;67(2):249-250.

13. Sayles PC, Wassom DL. Are antibodies important in mice infected with Plasmodium yoelii?. Parasitol Today. 1992;8(11):368-370.

14. Spencer LM, Quintana D, Hidalgo L. An assay to evaluate the inhibition of invasion in vitro of P. yoelii strain with different monoclonal antibodies raised against MSP-119. Biomédica [Internet]. 2008 [July 2020];19(1):45-51. Available from: https://dialnet. unirioja.es/servlet/articulo?codigo $=6058430$

15. Spencer LM, Ogun SA, Fleck SL, Ling IT, Scott-Finnigan TJ, Blackman $\mathrm{MJ}$, et al. Passive immunization with antibodies against three distinct epítopes on Plasmodium yoelii merozoite surface protein-1 suppresses parasitemia. Infect Immun. 1998;66(8):39253930.
16. Voller A, O'Neill P. Immunnofluorescence method suitable for large-scale application to malaria. Bull World Health Organ. 1971; 45(4):524-529.

17. Bidwell DE, Bartlett A, Voller A. Enzyme Immunoassays for Viral Diseases. J Infect Dis. October 1977;136(2): S274-S278.

18. Laemmli UK. Cleavage of structural proteins during the assembly of the head of bacteriophage T4. Nature. 1970;227(5259):680685.

19. Blackman MJ, Ling IT, Nicholls SC, Holder AA. Proteolytic processing of the Plasmodium falciparum merozoite surface protein-1 produces a membrane-bound fragment containing two epidermal growth factor-like domains. Mol Biochem Parasitol. 1991;49(1):29-33.

20.Pouvelle B, Fusaï T, Lépolard C, Gysin J. Biological and biochemical characteristics of cytoaghesion of Plasmodium falciparum infected erythrocytes to chondroitin-4-sulfate". Infect Immun. 1998;66(10):4950-4956.

21. Hasler T, Handunnetti SM, Aguiar JC, Schravendijk MR, Greenwood BM, et al. In vitro resetting, cytoadherence and microagglutination properties of Plasmodium falciparum infected erythrocytes from Gambian and Tanzanian patients. Blood. 1990;76(9):1845-1852.

22. IAEA (International Atomic Energy Agency). Radiation Biology: a handbook for teachers and students. Vol 42 [Internet]. Vienna, Austria: IAEA Publishing Section; 2010 [cited 2021 July 29]. Available from: https://www.iaea.org/publications/8219/radiation-biology-a-handbook-for-teachers-and-students

23. Holder AA, Freeman RR. Protective Antigens of Rodent and Human Bloodstage Malaria. Philos. Trans R Soc Lond B Biol Sci. 1984;307(1131):171-177.

24. McBride JS, Heidrich HG. Fragments of the polymorphic Mr 185 000 glycoprotein from the surface of isolated Plasmodium falciparum merozoites form an antigenic complex. Mol Biochem Parasitol. 1987;23(1):71-84.

25. Holder AA, Blackman MJ, Burghaus PA, Chappel JA, Ling IT, McCallum-Deighton $\mathrm{N}$, et al. A malaria merozoite surface protein (MSP1)-structure, processing and function. Mem Inst Oswaldo Cruz. 1992;87(3):37-42.

26. Chappel JA, Egan AF, Riley EM, Druilhe P, Holder AA. Naturally acquired human antibodies which recognize the first epidermal growth factor-like module in the Plasmodium falciparum merozoite surface protein 1 do nor inhibit parasite growth in vitro. Infect Immun. 1994;62(10): 4488-4494.

27. Rothlein R, Dustin ML, Marlin SD, Springer TA. A human intercellular adhesion molecule (ICAM-1) distinct from LFA-1. J Immunol. 1986;137(4):1270-1274.

28. Ho M, White NJ. Molecular mechanisms of cytoadherence in malaria. Am J Physiol Cell Physiol. 1999;276(6):C1231-C1242.

29. Claessens, A., Rowe, J.A. Selection of Plasmodium falciparum Parasites for Cytoadhesion to Human Brain Endothelial Cells. J Vis. 2012; 59:7-1.

30.Brown A, Turner L, Christoffersen S, Andrews KA, Szestak T, Zhao Y, Larsen S, Craig AG, Higgins MK. Molecular architecture of a complex between an adhesion protein from the malaria parasite and intracellular adhesion molecule 1. J Biol Chem. 2013; 288(8):5992-6003.

31. Lee WC, Russell B, Rénia L. Sticking for a cause: the falciparum malaria parasites cytoadherence paradigm. Front Immunol. 2019; 10:1444. 